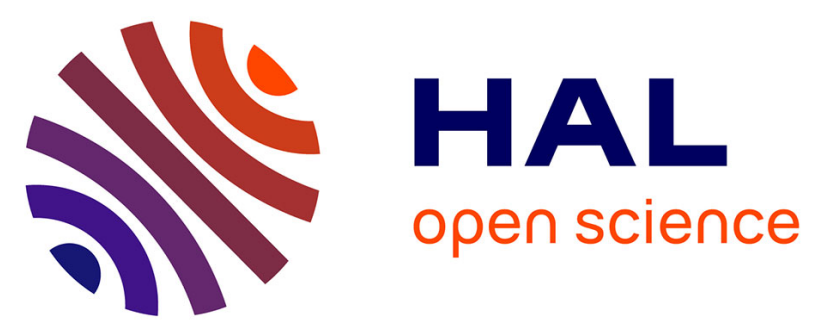

\title{
Night-sleep Duration Trajectories and Behavior in Preschoolers: Results from a Prospective Birth Cohort Study
}

Eve Reynaud, Anne Forhan, Barbara Heude, Marie-Aline Charles, Sabine Plancoulaine

\section{To cite this version:}

Eve Reynaud, Anne Forhan, Barbara Heude, Marie-Aline Charles, Sabine Plancoulaine. Night-sleep Duration Trajectories and Behavior in Preschoolers: Results from a Prospective Birth Cohort Study. Behavioral Sleep Medicine, 2020, pp.1-13. 10.1080/15402002.2020.1773467 . inserm-02872407

\section{HAL Id: inserm-02872407 https://www.hal.inserm.fr/inserm-02872407}

Submitted on 17 Jun 2020

HAL is a multi-disciplinary open access archive for the deposit and dissemination of scientific research documents, whether they are published or not. The documents may come from teaching and research institutions in France or abroad, or from public or private research centers.
L'archive ouverte pluridisciplinaire HAL, est destinée au dépôt et à la diffusion de documents scientifiques de niveau recherche, publiés ou non, émanant des établissements d'enseignement et de recherche français ou étrangers, des laboratoires publics ou privés. 
Night-sleep duration trajectories and behavior in preschoolers: results from a prospective birth cohort study

Eve Reynaud, $\mathrm{PhD}^{\mathrm{a}, \mathrm{b}}$; Anne Forhan ${ }^{\mathrm{b}}$; Barbara Heude, $\mathrm{PhD}^{\mathrm{b}}$; Marie-Aline Charles, $\mathrm{MD}^{\mathrm{b}}$ and Sabine Plancoulaine, $\mathrm{MD}^{\mathrm{b}}$

${ }^{\text {a }}$ CNRS UPR 3212, Team 9 - Institute of Cellular and Integrative Neurosciences (INCI)

15, rue du Général Rouvillois F-67000 Strasbourg, France

${ }^{\mathrm{b}}$ Université de Paris, CRESS, INSERM, INRAE U1153, F-75004 Paris, France

\section{Corresponding author}

Sabine Plancoulaine, INSERM U1153, Team 6 EAROH, 16 Avenue Paul Vaillant Couturier, 94807 Villejuif Cedex, France, [sabine.plancoulaine@inserm.fr], 33 145-595-109

\section{Presentation information}

These results were presented as a poster at the 2019 APSS-SLEEP meeting in San Antonio, TX, USA.

Word count: 3080 


\begin{abstract}
Background. Short sleep duration is associated with concomitant behavioral difficulties, but the longitudinal association between sleep duration and later behavior in early childhood remains unclear. The aim was to study the association between sleep duration trajectories throughout preschool and behavior at age 5-6 years.
\end{abstract}

Participants 1021 children of the French birth-cohort EDEN were included in this study.

Methods. Night-sleep duration trajectories were modeled on the basis of repeated measures collected at age 2, 3 and 5-6 years by parental questionnaires. Behavior was assessed with the Strengths and Difficulties Questionnaire (SDQ), with five subscales measuring conduct problems, emotional symptoms, peer relation problems, antisocial behavior, and hyperactivity-inattention problems. The subscales were dichotomized by the 25 th percentile. Multivariable logistic regression, adjusted for baseline behavior, and assessment of multiple confounding factors allowed for studying the independent association between night-sleep duration trajectories from age 2 to 6 and behavior at age 5-6.

Results. Five distinct sleep-duration trajectories were identified. At age 5-6 years, probability of hyperactivity-inattention problems was increased for boys belonging to the "short sleep" and "medium-low sleep" duration trajectory and girls belonging to the "changing sleep" duration trajectory as compared with children with the medium-high sleep duration trajectory (boys: OR 2.69 [95\% CI 1.18-6.16], $\mathrm{p}=0.03$ and 1.95 [1.25-3.03], $\mathrm{p}=0.003$, and girls: OR 2.79 [1.09 - 7.17], $\mathrm{p}=0.03)$. No associations were observed with the other SDQ subscales.

Conclusion. The persistence of short night-sleep durations in preschool years as well as a sudden decrease at age 3 are risk factors of hyperactivity-inattention problems at school start.

\title{
Keywords
}

Sleep duration, preschooler, behavior, group-based trajectory modeling, longitudinal study 


\section{Abbreviations}

SS: short-sleep duration trajectory

MLS: medium-low-sleep duration trajectory

MHS: medium-high-sleep duration trajectory

LS: long-sleep duration trajectory

CS: changing-sleep duration trajectory 


\section{Introduction}

Sleep duration and architecture evolve rapidly in the first years of life. The few studies that have modeled the evolution of sleep duration and sleep consolidation in early childhood revealed that this maturation varies among children (Plancoulaine et al., 2018; Reynaud et al., 2016; Touchette et al., 2009). Those studies also pointed out that without intervention, sleep difficulties, such as short sleep duration and frequent night-waking, show a persistent pattern from age 2 to 5-6 years.

A growing body of literature indicates that short sleep duration is associated with concomitant behavioral difficulties in school-age children (for review, see Astill, Van der Heijden, Van Ijzendoorn, \& Van Someren, 2012) but also as early as pre-school years (for review, see Reynaud, Vecchierini, Heude, Charles, \& Plancoulaine, 2018). Nonetheless, the longitudinal association between sleep duration and later behavior in preschool years is still an open topic of research, with few studies investigating this issue using a longitudinal design (BouvetteTurcot et al., 2015; Jansen et al., 2011; Sivertsen et al., 2015, 2015; Touchette et al., 2009; Williams et al., 2017). In the Jansen et al. study (2011), which included 4782 children, sleep duration at age 2 years was associated with anxious/depressed symptoms at age 3 years, and this association remained significant after adjusting for baseline anxious/depressed symptoms (at 18 months). Bouvette-Turcot et al. (2015) observed a genetic susceptibility in the longitudinal association between sleep duration and behavior: average sleep duration from 6 to 36 months was negatively associated with emotionality/behavioral dysregulation at age 36 months in the 131 children with one or two copies of the short allele of the serotonin transporter gene (5-HTTLPR) but not for other children. These studies focused on associations with a single internalizing behavior, but Siverstsen et al. (2015) covered a larger range of behavioral aspects among 32662 children: as compared with children who slept $\geq 13$ $\mathrm{hr}$ at 18 months, those who slept $\leq 10 \mathrm{hr}$ were significantly more likely to present higher 
aggressive behavior, attention problems, anxiety/depression and emotional reactivity at age 5 years; the difference with somatic complaints was not significant. Two studies considered several exposure points of sleep duration in preschool years. In the Huhdanpää et al. study (2019), sleep duration at 3 months, 8 months and 24 months was studied separately and associated with inattention and hyperactivity at age 5 years. All time points considered individually were negatively associated with later inattention but none were associated with later hyperactivity. In the Touchette et al. study (2009), sleep trajectories from age 1.5 to 5 years were modeled in 2056 children using group-based trajectory methodology (GBTM). This method optimizes the use of the information obtained from repeated measures and allows for complex evolution modeling. The authors showed increased risk of hyperactivity in children presenting persistent short sleep duration between age 1.5 and 5 years (i.e., $\leq 9$ hr/night). No study has reported the longitudinal evolution (as identified by GBTM) and several behavioral dimensions in the same population.

To complete the current literature, we assessed the association of night-sleep duration trajectories from age 2 to 5-6 (modeled using GBTM) with multiple facets of behavior at age 5-6, in a large birth-cohort. Our hypothesis was that sleep trajectories, which better describe sleep duration evolution over time, may predict behavioral difficulties in preschoolers.

\section{Methods}

\section{Study population}

Details of the study protocol have been published previously (Heude et al., 2015). To summarize, pregnant women before their 24th week of amenorrhea were recruited between 2003 and 2006 in two French university hospitals (Poitiers and Nancy). Those younger than 18, unable to give informed consent, functionally illiterate in French, with a history of diabetes, planning to change residence or without social security coverage were excluded 
from the cohort. Women with multiple pregnancies were also excluded. Among the 3758 women invited to participate, 2002 (53\%) agreed to participate. Finally, because of miscarriages, stillbirths, and attrition, 1899 children were included at birth. Written informed consent was obtained twice from parents: at enrolment and after the child's birth. The study was approved by the ethics research committee of Bicêtre Hospital (Comité Consultatif de Protection des Personnes dans la Recherche Biomédicale) and by the Data Protection Authority (Commission Nationale de l'Informatique et des Libertés).

\section{Measures}

All variables included in the analysis were collected by paper-based parent questionnaires. Measure of exposure: night-sleep duration trajectory.

When the child was age 2, 3, and 5-6 years, parents were asked about the usual bedtime and wake-up time of the child, which allowed for estimating the nighttime-in-bed duration at each age. To optimize the analysis of this longitudinal data, we modeled the nighttime-in-bed sleep duration evolution over time. Repeated measures were used to model a proxy of night-sleep duration trajectories across preschool years among the 1205 children with sufficient data (i.e., at least two time points of night-sleep duration) by using group-based trajectory modeling (Nagin, 2005). The method is based on the underlying hypothesis that, within a population, inherent groups evolve according to different patterns. The groups are not directly identifiable or pre-established by sets of characteristics but are statistically determined by series of responses. Using the PROC TRAJ procedure in SAS 9.4 (SAS Institute Inc., Cary, NC, USA), multiple models were created, varying by number of groups and shapes (computed by polynomial equations). The best-fitted model was determined by comparing those models by using, among other criteria, Bayesian information criteria (BIC). Its quality was verified according to the recommended criteria: average posterior probabilities for each subgroup > 0.7 , odds of correct classification $>5$, and the model's estimation of the trajectory prevalence 
and the actual prevalence being close ( $\leq 3 \%$ difference) (Nagin, 2005). Each child was attributed to the trajectory for which he/she had the highest probability of belonging to.

Five night-sleep duration trajectories were identified: a short-sleep (SS) duration trajectory (<10 hr $30 \mathrm{~min} /$ night) representing $5.0 \%$ of children; a medium-low-sleep (MLS) duration trajectory (10 hr $30 \mathrm{~min}$ to $11 \mathrm{hr} /$ night) representing $48.4 \%$ of children; a medium-high-sleep (MHS) duration trajectory (about $11 \mathrm{hr} 30 \mathrm{~min} /$ night) representing 37.0\% of children; a longsleep (LS) duration trajectory ( $\geq 11 \mathrm{hr} 30 \mathrm{~min} /$ night) representing $4.3 \%$ of children; and a changing-sleep (CS) duration trajectory (i.e., up to age 3 similar to the LS and then MLS duration trajectory) representing $5.3 \%$ of children (Figure 1). Further descriptions of the trajectories were previously published (Plancoulaine et al., 2018).

\section{Measure of outcome: behaviors}

Behavior was assessed by using the parent version of the Strengths and Difficulties Questionnaire (SDQ) (Goodman, 1997) when the child was on average 5.6 years old (SD= 0.16). The SDQ includes five subscales ranging from 0 to 10: four subscales assess behavioral problems (hyperactivity-inattention, conduct problems, emotional symptoms, and peer relationship problems) and one subscale assesses pro-social behavior. In subscales assessing behavioral problems, a higher score indicates greater difficulties, with the reverse for the prosocial subscale. The subscale scores were skewed and could not be normalized, so they were dichotomized based on the studied population distribution. For each behavioral problem subscale, children were divided into those with a score greater than the 75 th percentile and the others. This threshold was considered to allow sufficient sample size in each category. For the pro-social subscale, the division was between those with a score lower than the 25th percentile and the others, thus favoring identification of the highest behavioral difficulties within the studied population. This dichotomization corresponded to a score $>4$ for 
hyperactivity-inattention, $>3$ for conduct problems, $>3$ for emotional symptoms, $>1$ for peer relationship problems, and $<8$ for pro-social behavior. Thus, each behavior outcome corresponded to the question: "Is the child among the $25 \%$ of children with the highest behavioral difficulty score?" (yes/no). On sensitivity analysis, the analyses were repeated by using the clinical dichotomization thresholds validated in French school-aged children (Shojaei et al., 2009): >5 for hyperactivity-inattention, $>2$ for conduct problems, $>3$ for emotional symptoms, $>3$ for peer relationship problems, and $<7$ for pro-social behavior. Each behavior outcome corresponded to the question: "Does the child have a behavioral difficulty score above the norm?" (yes/no). Emotional symptoms, hyperactivity-inattention problems, conduct problems, and pro-social behaviors were also evaluated at age 2 by using a parental behavioral questionnaire specific to the EDEN birth cohort study (Table S3).

\section{Covariates}

Covariates were integrated in the statistical models to take into account potential confounding factors, including parental and child characteristics and lifestyle. Their selection was based on the literature (Reynaud et al., 2018) and bivariate analyses (i.e., age-adjusted associations between SDQ subscale scores and covariates, Table S1).

Covariates regarding parental characteristics collected during pregnancy included the following:

- Household income $(<1500 €, 1500-3000 €,>3000 €$ per month; US dollar equivalent: $<\$ 1600$, $\$ 1600-3250,>\$ 3250$ per month)

- Education (defined as the highest number of years of study reached by one of two parents)

- Mother's age at delivery

- Smoking during pregnancy (yes/no)

- History of parental behavior problems in childhood (one or both parents declaring that they 
had a behavioral problem during childhood)

- Mother's depressive symptoms during pregnancy (evaluated with the Center of Epidemiologic Studies Depression scale (CES-D), with a cutoff score of 23, threshold validated in French women (Fuhrer \& Rouillon, 1989)).

Covariates regarding the child's characteristics included the following:

$-\operatorname{Sex}$

- Birth order (first/other)

- Term at birth (weeks of amenorrhea)

- Breastfeeding duration (in months)

- Temperamental traits (namely activity, shyness, emotionality and sociability, assessed at age 1 year by using the Emotionality Activity and Sociability scale (EAS) (Buss \& Plomin, 2008))

Covariates regarding life style and sleep habits at age 2 years included the following:

- Maternal working status (yes/no)

- Number of hours spent in front of the television per day (collected separately for school days and free days from which we deduced an average time per day).

- Main care arrangement (collective settings such as preschool or day care centers vs home care)

- Parental presence needed for the child to fall asleep (yes/no)

- Child regular bedtime (yes/no)

- Child frequent night-waking defined as occurring every other night or more per week (yes/no) (Reynaud et al., 2016)

- Nap duration (hours per day) 


\section{Statistical Analysis}

Missing data for the covariates represented $3.3 \%$ of the total dataset and multiple imputations were performed by using chained equations (SAS "MI" procedure). Variables were imputed by fully conditional specification methods (i.e., using discriminant, logistic or linear regression methods according to the variable types). We generated 10 independent imputed datasets.

The SAS Mianalyze procedure was used for multivariable logistic regression analysis of the associations between the night-sleep duration trajectories and each of the five SDQ behavior subscales (in their dichotomized form, described above). First, we analyzed the raw association between night-sleep duration trajectories and behavior at age 5-6 (Table 2, raw model), then adjusted for potential confounding factors described above (Table 2, model 1). Next, we adjusted additionally for the corresponding behavior at age 2 (Table 2, model 2) to take into account baseline behavior and account for potential reverse causality. Model 2 was not performed for the peer-relationship problem subscale because it was not evaluated in the birth-cohort at age 2 years. Sex interaction was investigated between sleep duration trajectories and behavior. Analyses with the final models were repeated by using the clinical thresholds for all SDQ subscales, validated in French school-aged children, as sensitivity analyses.

Statistical analyses were performed with SAS 9.4 (SAS Institute Inc., Cary, NC, USA). $\mathrm{P}<0.05$ was considered statistically significant.

\section{Results}

\section{Population Description}

Information regarding night-sleep duration trajectory and behavior was available for 1021 children, representing $54 \%$ of the children enrolled at birth. As compared with children who 
were not included, for those who were included, parents had a higher income $(30.0 \%$ vs $23.4 \%$ had income $>3000 €$ per month, $\mathrm{p}<0.0001)$ and higher level of education (14.6 vs 13.4 years of study, $\mathrm{p}<0.001)$. Mothers were slightly older $(30.2$ vs 28.7 years, $\mathrm{p}<0.001)$, less depressed (6.0\% vs $12.1 \%, \mathrm{p}<0.001)$, smoked less during pregnancy (19.9\% vs $32.0 \%$, $\mathrm{p}<0.001)$ and breastfed for longer (3.4 vs 3.0 months, $\mathrm{p}=0.02)$.

The characteristics of the 1021 children included in the analysis are in Table 1. Mothers were 30 years old on average at delivery and $46 \%$ of the children were girls. At age 2 , children watched 41 min of television per day on average.

[Table 1 near here]

\section{Associations}

The raw and adjusted odds ratios (aORs) between night-sleep duration trajectories and child behavior are presented in Table 2. Sleep duration trajectories were significantly associated with hyperactivity-inattention in both models. Thus, SS, MLS and CS trajectories were associated with increased probability of hyperactivity-inattention at age 5-6 independent of hyperactivity-inattention behavior at age 2. We observed no association between sleep duration trajectories and the other subscales, namely conduct problems, emotional symptoms, peer relationship problems and low pro-social behavior subscales.

[Table 2 near here]

For conduct problems, hyperactivity-inattention and low pro-social behavior subscales, probability of being among the $25 \%$ of children with the highest problematic behavior was greater for boys than girls (Table 3). We observed an interaction between sleep trajectories and hyperactivity-inattention $(\mathrm{p}=0.07)$ but not other subscales.

[Table 3 near here]

Probability of hyperactivity-inattention at age 5-6 was increased for boys in the SS and MLS 
duration trajectories, but no associations were found for the CS duration trajectory (Table 4). However, probability of hyperactivity-inattention at age 5-6 was increased for girls in the CS duration trajectory (Model 2 aOR 2.83 p=0.03), with no associations found for the SS or MLS duration trajectories.

On sensitivity analysis, with SDQ clinical thresholds used instead of thresholds based on the sample distribution, fewer children were classified as having hyperactivity-inattention $(\mathrm{N}=159 ; 106$ boys). The association between the sleep trajectories and this subscale was similar for boys but was not significant for girls (Table S2).

[Table 4 near here]

\section{Discussion}

This study showed that persistent night-sleep duration $<11 \mathrm{hr} /$ night between age 2 and 5-6 years is associated with increased risk of hyperactivity-inattention symptoms evaluated by the SDQ at age 5-6 years, independent of hyperactivity-inattention at age 2 . We found no associations between night-sleep duration trajectories and other SDQ subscales.

In the only other longitudinal study that focused on associations between night-sleep duration and hyperactivity, results were consistent with ours. Indeed, in a sample of 2056 children aged 1.5 to 5 years, Touchette et al. (2009) showed that persistent short sleepers ( $\leq 9 \mathrm{hr} / \mathrm{night})$ presented a 4-fold increased risk of hyperactivity. The SDQ hyperactivity-inattention subscale has been found an accurate tool for the screening of attention deficit hyperactivity disorder (ADHD) in preschoolers when rated by parents and using a threshold of $>4$ (Øvergaard et al., 2018), as in the current study.

Unlike Jansen et al. (2011), we did not find an association between sleep duration and internalizing symptoms. However, we assessed internalizing symptoms by using the emotional symptoms and the peer-relationship problems subscale of the SDQ, whereas Jansen et al. used the anxiety/depressed symptoms subscale of the Child Behavior Checklist. In 
addition, the two studies used different timing for both sleep duration and internalizing symptoms measures. We analyzed persistent night sleep durations between ages 2 and 5-6 years, adjusted on day sleep, in relation with internalizing symptoms measured at age 5-6 years, whereas Jansen et al. analyzed mean total sleep duration at age 2 years (including day sleep) in relation with internalizing symptoms at age 3 years. These differences in age, tool and dimension measured could explain the discrepancy.

Our results indicated greater behavioral difficulties among boys than girls, which is consistent with the literature, especially for hyperactivity-inattention behavior; indeed, ADHD prevalence has been found to be two to three times higher in boys than girls (Polanczyk et al., 2007; Romano et al., 2006). Also, boys show shorter sleep duration than do girls (Biggs et al., 2013; Blair et al., 2012; Plancoulaine et al., 2015). In a recent cross-sectional study of 181 US children (125 boys) aged 5-13 years, girls with ADHD showed longer sleep duration but more sleep disturbances than did boys (Becker et al., 2018), which provides preliminary evidence of sex differences in sleep functioning among children with ADHD. In our population sample, after adjustment on child temperament and behavior evaluation at age 2 years, only boys in the two persistent shortest night sleep duration trajectories (i.e., $<10 \mathrm{hr} 30 \mathrm{~min}$ and $10 \mathrm{hr} 30$ min to $11 \mathrm{hr} /$ night between age 2 and 5-6 years) showed increased probability of hyperactivity-inattention score at age 5-6, with a dose effect (aOR 2.69 [95\% CI 1.18-6.16] and 1.95 [1.25-3.03]). Only girls belonging to the CS duration trajectory (i.e., $\geq 11 \mathrm{hr} 30$ min/night up to 3 years and about $10 \mathrm{hr} 30 \mathrm{~min}$ to $11 \mathrm{hr} /$ night thereafter) showed increased probability of hyperactivity-inattention score at age 5-6 years (aOR 2.79 [1.09-7.17]). The prevalence of reported hyperactivity-inattention is lower in girls than boys ( $20.4 \%$ vs $32.0 \%$ ), so the lack of associations observed between the shorter sleep duration trajectories (SS and MLS) and hyperactivity-inattention in this group may result from low statistical power rather than true sex differences. This hypothesis is supported by the ORs being quite close for both 
sexes. However, the association between the CS duration trajectory and hyperactivityinattention is specific to girls. Hence, for girls, the observed effect seems linked to the abrupt decrease in sleep duration between age 2 and 3 years, but whether there is also a role of prolonged short sleep duration throughout the preschool years is unclear. Observed differences between boys and girls could be due to rating scales and some lab tests showing that girls have a lower base level of hyperactivity-inattention than do boys, and thus, "they have to deviate farther from sex peers than do boys to attain a "diagnostic" level of problems" (Arnold, 1996) (page 559). Also, girls with ADHD are seemingly less diagnosed than are boys (Mowlem et al., 2019). One of the explanations proposed by Quinn and Madhoo (2014) is that girls with ADHD more often show the inattentive form of ADHD than do boys, which can be perceived as less problematic as compared with disruptive behavior. In addition, parental evaluation and reports could differ depending on the child's sex. Indeed, girls are less likely to be referred to specialized medical centers for ADHD than are boys. This difference was partly explained by parents believing that learning assistance is less effective for girls than boys with ADHD (Ohan et al., 2009).

The main strength of this study was the use of the trajectory-modeling method to take into account the longitudinal aspects of sleep rather than a single time point. Also, by taking into account baseline behavior, we could show a temporality in the association between sleep duration and hyperactivity-inattention problems. Of note, the results do not discount the possibility of remaining reverse associations (i.e., pre-existing behavioral difficulties before age 2 years and subsequent short sleep duration) (Gruber, 2014; Sadeh, 2007; Touchette et al., 2009) nor the possibility for sleep to be an inherent trait of external behavior problems such as ADHD (Bijlenga et al., 2019). The rationale for using group-based trajectory modeling is that although classical longitudinal studies (analyzing the association between a given exposure and an ulterior outcome) allow for establishing the temporality of the association, they are not 
optimal for the study of repeated measures, because they consider only one time of exposure per analysis. Repeating the analysis for each time point is feasible, but the evolution of the exposure through time is not taken into account; thus, for each given time point, a transient exposure is considered equivalent to a long-lasting one. Here we used a data-driven strategy that allows for identifying several groups of children with similar patterns that could be potential specific targets for counseling and prevention. Confounding bias was limited with the inclusion of a wide range of covariates, established from a systematic review of the literature (Reynaud et al., 2018) combined with a preliminary analysis.

Structural equation modeling has also been used to study repeated measures of sleep associated with behavioral difficulties. In the Williams et al. study (2017), which included more than 4000 children assessed at five time points from age 0-1 year to 8-9 years, behavioral sleep problems were consistently associated with increased emotional dysregulation 2 years later and vice versa. In the Mindell et al. study (2017), structural equation modeling indicated that sleep (defined by a latent variable composed of sleep duration and bed time) at 12 months was associated with internalizing behavior at age 18 months. However the reverse association (pre-existing internalizing behavior and later sleep difficulties) was not found.

Several limitations of this study should be noted. The first is the subjective aspects of the measures. Parents reported time in bed duration, which possibly led to an overestimation of the real night-sleep duration. Assessment of the child's behavior involved the parental version of the SDQ, which has good external validation (Croft et al., 2015; Shojaei et al., 2009) and detection power for internalizing and externalizing behavior (Goodman \& Scott, 1999). Confounding factors were also subjectively assessed, so some of the confounding effect still remained in the analyses. Another limitation is the relatively small sample size, which allowed for the required statistical power to investigate our main hypothesis but not the sex 
specificity. Also, the sample is not representative of the general French population because of the initial recruitment (mainly urban and based on voluntary participation) and also attrition (classically, women with more education and income were more likely to remain in the study). This limits the generalization of the results. Finally, the role of chronotype could not be investigated because it was not measured in the cohort. This would have been of interest because chronotype may have a preponderant role in school-aged children, with evening chronotypes showing increased risk for behavioral problems (van der Heijden et al., 2013).

An etiological hypothesis for the longitudinal association between sleep and hyperactivityinattention is that sleep regulates synaptic homeostasis, so lack of sleep could reduce synaptic plasticity (Tononi \& Cirelli, 2014) and have behavioral repercussions such as reduced capacity to adapt to the environment (Kolb \& Gibb, 2014). Also, brain maturation and synaptic plasticity related to sleep may differ by sex in typically developing infants and children (Franco et al., 2020).

This study, combined with previous literature on the topic, brings additional knowledge regarding predictors of hyperactivity-inattention in early childhood, which is especially important since they are predictive of academic under-achievement at age 12 (Salla et al., 2016). We need a better understanding of the etiology of behavioral difficulties to improve medical care in this regard and better target prevention programs. Although some sleep intervention programs have shown promising results for reducing ADHD symptoms (Hiscock et al., 2015), current reviews of treatment strategies do not mention sleep as a nonpharmacological option (Caye et al., 2019).

In conclusion, night-sleep duration trajectories in early years are positively associated with hyperactivity-inattention problems at school start but not with other behavioral features. In our sample, associations were sex-dependent, but additional studies are needed to confirm these results. 


\section{Acknowledgments}

We thank the EDEN mother-child cohort study group (I. Annesi-Maesano, J.Y Bernard, J. Botton, M.A. Charles, P. Dargent-Molina, B. de Lauzon-Guillain, P. Ducimetière, M. de Agostini, B. Foliguet, A. Forhan, X. Fritel, A. Germa, V. Goua, R. Hankard, B. Heude, M. Kaminski, B. Larroque†, N. Lelong, J. Lepeule, G. Magnin, L. Marchand, C. Nabet, F. Pierre, R. Slama, M.J. Saurel-Cubizolles, M. Schweitzer, O. Thiebaugeorges).

We thank all funding sources for the EDEN study (not allocated for the present study but for the cohort): Foundation for medical research (FRM), National Agency for Research (ANR), National Institute for Research in Public health (IRESP: TGIR cohorte santé 2008 program), French Ministry of Health (DGS), French Ministry of Research, INSERM Bone and Joint Diseases National Research (PRO-A) and Human Nutrition National Research Programs, Paris-Sud University, Nestlé, French National Institute for Population Health Surveillance (InVS), French National Institute for Health Education (INPES), the European Union FP7 programs (FP7/2007-2013, HELIX, ESCAPE, ENRIECO, Medall projects), Diabetes National Research Program (in collaboration with the French Association of Diabetic Patients (AFD), French Agency for Environmental Health Safety (now ANSES), Mutuelle Générale de l'Education Nationale complementary health insurance (MGEN), French national agency for food security, French speaking association for the study of diabetes and metabolism (ALFEDIAM). The funding source had no involvement in the conception of the present study.

\section{Declaration of interest}

Authors have no declaration of interest to declare 


\section{References}

Arnold, L. E. (1996). Sex differences in ADHD: Conference summary. Journal of Abnormal Child Psychology, 24(5), 555-569. https://doi.org/10.1007/BF01670100

Astill, R. G., Van der Heijden, K. B., Van IJzendoorn, M. H., \& W, J. (2012). Sleep, cognition, and behavioral problems in school-age children: A century of research meta-analyzed. Psychological Bulletin, 138(6), 1109-1138. https://doi.org/10.1037/a0028204

Becker, S. P., Cusick, C. N., Sidol, C. A., Epstein, J. N., \& Tamm, L. (2018). The impact of comorbid mental health symptoms and sex on sleep functioning in children with ADHD. European Child \& Adolescent Psychiatry, 27(3), 353-365. https://doi.org/10.1007/s00787-017-1055-2

Biggs, S. N., Lushington, K., Martin, A. J., van den Heuvel, C., \& Kennedy, J. D. (2013). Gender, socioeconomic, and ethnic differences in sleep patterns in school-aged children. Sleep Medicine, 14(12), 1304-1309. psyh. https://doi.org/10.1016/j.sleep.2013.06.014

Bijlenga, D., Vollebregt, M. A., Kooij, J. J. S., \& Arns, M. (2019). The role of the circadian system in the etiology and pathophysiology of ADHD: Time to redefine ADHD? Attention Deficit and Hyperactivity Disorders, 11(1), 5-19. https://doi.org/10.1007/s12402-018-0271-z

Blair, P. S., Humphreys, J. S., Gringras, P., Taheri, S., Scott, N., Emond, A., Henderson, J., \& Fleming, P. J. (2012). Childhood sleep duration and associated demographic characteristics in an English cohort. Sleep, 35(3), 353-360. https://doi.org/10.5665/sleep.1694

Bouvette-Turcot, A.-A., Pluess, M., Bernier, A., Pennestri, M.-H., Levitan, R., Sokolowski, M. B., Kennedy, J. L., Minde, K., Steiner, M., Pokhvisneva, I., Meaney, M. J., Gaudreau, H., \& MAVAN research team. (2015). Effects of Genotype and Sleep on Temperament. Pediatrics, 136(4), e914-921. https://doi.org/10.1542/peds.2015-0080

Buss, A. h., \& Plomin, R. (2008). Temperament: Early developing personality traits (3rd ed.). Psychology press.

Caye, A., Swanson, J. M., Coghill, D., \& Rohde, L. A. (2019). Treatment strategies for ADHD: An evidence-based guide to select optimal treatment. Molecular Psychiatry, 24(3), 390-408. https://doi.org/10.1038/s41380-018-0116-3 
Croft, S., Stride, C., Maughan, B., \& Rowe, R. (2015). Validity of the strengths and difficulties questionnaire in preschool-aged children. Pediatrics, 135(5), e1210-1219. https://doi.org/10.1542/peds.2014-2920

Franco, P., Putois, B., Guyon, A., Raoux, A., Papadopoulou, M., Guignard-Perret, A., Bat-Pitault, F., Hartley, S., \& Plancoulaine, S. (2020). Sleep during development: Sex and gender differences. Sleep Medicine Reviews, 51, 101276. https://doi.org/10.1016/j.smrv.2020.101276

Fuhrer, R., \& Rouillon, F. (1989). La version française de l'échelle CES-D (Center for Epidemiologic Studies-Depression Scale). Description et traduction de l'échelle d'auto-évaluation. Psychiatrie et Psychobiologie, 163-166.

Goodman, R. (1997). The Strengths and Difficulties Questionnaire: A research note. Journal of Child Psychology and Psychiatry, and Allied Disciplines, 38(5), 581-586.

Goodman, R., \& Scott, S. (1999). Comparing the Strengths and Difficulties Questionnaire and the Child Behavior Checklist: Is small beautiful? Journal of Abnormal Child Psychology, 27(1), $17-24$.

Gruber, R. (2014). ADHD, anxiety and sleep: A window to understanding the interplay between sleep, emotional regulation and attention in children? Behavioral Sleep Medicine, 12(1), 84-87. https://doi.org/10.1080/15402002.2014.862089

Heude, B., Forhan, A., Slama, R., Douhaud, L., Bedel, S., Saurel-Cubizolles, M.-J., Hankard, R., Thiebaugeorges, O., De Agostini, M., Annesi-Maesano, I., Kaminski, M., Charles, M.-A., \& EDEN mother-child cohort study group. (2015). Cohort Profile: The EDEN mother-child cohort on the prenatal and early postnatal determinants of child health and development. International Journal of Epidemiology. https://doi.org/10.1093/ije/dyv151

Hiscock, H., Sciberras, E., Mensah, F., Gerner, B., Efron, D., Khano, S., \& Oberklaid, F. (2015). Impact of a behavioural sleep intervention on symptoms and sleep in children with attention deficit hyperactivity disorder, and parental mental health: Randomised controlled trial. BMJ: British Medical Journal, 350. psyh. https://frodon.univparis5.fr/url?http://search.ebscohost.com/login.aspx?direct=true\&db=psyh\&AN=2015-07614001\&lang=fr\&site=ehost-live 
Huhdanpää, H., Morales-Muñoz, I., Aronen, E. T., Pölkki, P., Saarenpää-Heikkilä, O., Paunio, T., Kylliäinen, A., \& Paavonen, E. J. (2019). Sleep Difficulties in Infancy Are Associated with Symptoms of Inattention and Hyperactivity at the Age of 5 Years: A Longitudinal Study. Journal of Developmental and Behavioral Pediatrics: JDBP, 40(6), 432-440. https://doi.org/10.1097/DBP.0000000000000684

Jansen, P. W., Saridjan, N. S., Hofman, A., Jaddoe, V. W. V., Verhulst, F. C., \& Tiemeier, H. (2011). Does disturbed sleeping precede symptoms of anxiety or depression in toddlers? The generation R study. Psychosomatic Medicine, 73(3), 242-249. https://doi.org/10.1097/PSY.0b013e31820a4abb

Kolb, B., \& Gibb, R. (2014). Searching for the principles of brain plasticity and behavior. Cortex; $a$ Journal Devoted to the Study of the Nervous System and Behavior, 58, 251-260. https://doi.org/10.1016/j.cortex.2013.11.012

Mindell, J. A., Leichman, E. S., DuMond, C., \& Sadeh, A. (2017). Sleep and Social-Emotional Development in Infants and Toddlers. Journal of Clinical Child and Adolescent Psychology: The Official Journal for the Society of Clinical Child and Adolescent Psychology, American Psychological Association, Division 53, 46(2), 236-246. https://doi.org/10.1080/15374416.2016.1188701

Mowlem, F. D., Rosenqvist, M. A., Martin, J., Lichtenstein, P., Asherson, P., \& Larsson, H. (2019). Sex differences in predicting ADHD clinical diagnosis and pharmacological treatment. European Child \& Adolescent Psychiatry, 28(4), 481-489. https://doi.org/10.1007/s00787$018-1211-3$

Nagin, D. (2005). Group-based modeling of development. Harvard University Press.

Ohan, J. L., \& Visser, T. A. W. (2009). Why is there a gender gap in children presenting for attention deficit/hyperactivity disorder services? Journal of Clinical Child and Adolescent Psychology: The Official Journal for the Society of Clinical Child and Adolescent Psychology, American Psychological Association, Division 53, 38(5), 650-660. https://doi.org/10.1080/15374410903103627 
Øvergaard, K. R., Oerbeck, B., Friis, S., Pripp, A. H., Biele, G., Aase, H., \& Zeiner, P. (2018).

Attention-Deficit/Hyperactivity Disorder in Preschoolers: The Accuracy of a Short Screener. Journal of the American Academy of Child \& Adolescent Psychiatry, 57(6), 428-435. https://doi.org/10.1016/j.jaac.2018.03.008

Plancoulaine, S., Lioret, S., Regnault, N., Heude, B., Charles, M.-A., \& the Eden Mother-Child Cohort Study Group. (2015). Gender-specific factors associated with shorter sleep duration at age 3 years. Journal of Sleep Research, 24(6), 610-620. https://doi.org/10.1111/jsr.12308

Plancoulaine, S., Reynaud, E., Forhan, A., Lioret, S., Heude, B., Charles, M.-A., Annesi-Maesano, I., Bernard, J. Y., Botton, J., Charles, M. A., Dargent-Molina, P., de Lauzon-Guillain, B., Ducimetière, P., de Agostini, M., Foliguet, B., Forhan, A., Fritel, X., Germa, A., Goua, V., ... Thiebaugeorges, O. (2018). Night sleep duration trajectories and associated factors among preschool children from the EDEN cohort. Sleep Medicine, 48, 194-201. https://doi.org/10.1016/j.sleep.2018.03.030

Polanczyk, G., de Lima, M., Horta, B., Biederman, J., \& Rohde, L. (2007). The worldwide prevalence of ADHD: A systematic review and metaregression analysis. American Journal of Psychiatry, 164(6), 942-948.

Quinn, P. O., \& Madhoo, M. (2014). A Review of Attention-Deficit/Hyperactivity Disorder in Women and Girls: Uncovering This Hidden Diagnosis. The Primary Care Companion for CNS Disorders, 16(3). https://doi.org/10.4088/PCC.13r01596

Reynaud, E., Forhan, A., Heude, B., de Lauzon-Guillain, B., Charles, M.-A., \& Plancoulaine, S. (2016). Night-waking trajectories and associated factors in French preschoolers from the EDEN birth-cohort. Sleep Medicine, 27-28, 59-65.

https://doi.org/10.1016/j.sleep.2016.09.008

Reynaud, E., Vecchierini, M.-F., Heude, B., Charles, M.-A., \& Plancoulaine, S. (2018). Sleep and its relation to cognition and behaviour in preschool-aged children of the general population: A systematic review. Journal of Sleep Research, 27(3), e12636.

https://doi.org/10.1111/jsr.12636 
Romano, E., Tremblay, R. E., Farhat, A., \& Côté, S. (2006). Development and prediction of hyperactive symptoms from 2 to 7 years in a population-based sample. Pediatrics, 117(6), 2101-2110. https://doi.org/10.1542/peds.2005-0651

Sadeh, A. (2007). Consequences of Sleep Loss or Sleep Disruption in Children. Sleep Medicine Clinics, 2(3), 513-520. https://doi.org/10.1016/j.jsmc.2007.05.012

Salla, J., Michel, G., Pingault, J. B., Lacourse, E., Paquin, S., Galéra, C., Falissard, B., Boivin, M., Tremblay, R. E., \& Côté, S. M. (2016). Childhood trajectories of inattention-hyperactivity and academic achievement at 12 years. European Child \& Adolescent Psychiatry, 25(11), 11951206. https://doi.org/10.1007/s00787-016-0843-4

Shojaei, T., Wazana, A., Pitrou, I., \& Kovess, V. (2009). The strengths and difficulties questionnaire: Validation study in French school-aged children and cross-cultural comparisons. Social Psychiatry and Psychiatric Epidemiology, 44(9), 740-747. http://dx.doi.org.ezaccess.libraries.psu.edu/10.1007/s00127-008-0489-8

Sivertsen, B., Harvey, A. G., Reichborn-Kjennerud, T., Torgersen, L., Ystrom, E., \& Hysing, M. (2015). Later emotional and behavioral problems associated with sleep problems in toddlers: A longitudinal study. JAMA Pediatrics, 169(6), 575-582. https://doi.org/10.1001/jamapediatrics.2015.0187

Tononi, G., \& Cirelli, C. (2014). Sleep and the price of plasticity: From synaptic and cellular homeostasis to memory consolidation and integration. Neuron, 81(1), 12-34. https://doi.org/10.1016/j.neuron.2013.12.025

Touchette, E., Côté, S. M., Petit, D., Liu, X., Boivin, M., Falissard, B., Tremblay, R. E., \& Montplaisir, J. Y. (2009). Short nighttime sleep-duration and hyperactivity trajectories in early childhood. Pediatrics, 124(5), e985-993. https://doi.org/10.1542/peds.2008-2005

van der Heijden, K. B., de Sonneville, L. M. J., \& Swaab, H. (2013). Association of eveningness with problem behavior in children: A mediating role of impaired sleep. Chronobiology International, 30(7), 919-929. https://doi.org/10.3109/07420528.2013.790041

Williams, K. E., Berthelsen, D., Walker, S., \& Nicholson, J. M. (2017). A Developmental Cascade Model of Behavioral Sleep Problems and Emotional and Attentional Self-Regulation Across 
Early Childhood. Behavioral Sleep Medicine, 15(1), 1-21.

https://doi.org/10.1080/15402002.2015.1065410 


\section{Figures legend}

Figure 1. Night sleep duration trajectories obtained among the preschool children of the French birth-cohort. Black triangles $=$ Short sleepers $(\mathrm{SS}, 5.0 \%$ of the children $)$, squares = Medium Low sleepers (MLS, 48.4\% of the children), circles = Medium High sleepers (MHS, $37.0 \%$ of the children $)$, white squares $=$ Changing $(\mathrm{CS}, 5.3 \%$ of the children $)$ and diamonds $=$

Long sleepers (LS, $4.3 \%$ of the children). Lines represent mean sleep duration trajectories. 


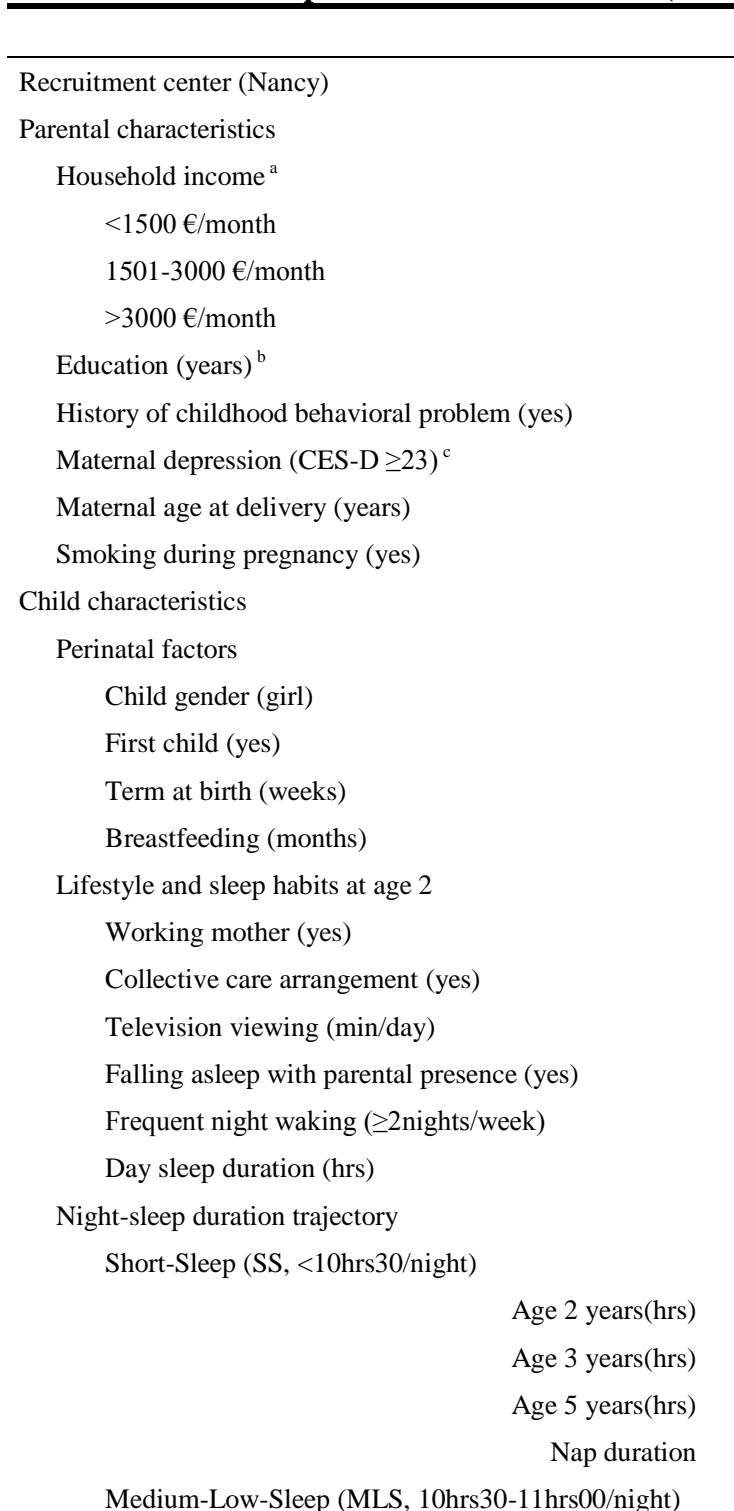

Medium-Low-Sleep (MLS, 10hrs30-11hrs00/night)

Age 2 years(hrs)

Age 3 years(hrs)

Age 5 years(hrs)

Nap duration

Medium-High-Sleep (MHS, $\approx 11 \mathrm{hrs} 30 /$ night)

$$
\begin{array}{r}
\text { Age } 2 \text { years(hrs) } \\
\text { Age } 3 \text { years(hrs) } \\
\text { Age } 5 \text { years(hrs) } \\
\text { Nap duration }
\end{array}
$$

Changing-Sleep (CS, LS up to 3y then MLS)

$$
\begin{aligned}
& \text { Age } 2 \text { years(hrs) } \\
& \text { Age } 3 \text { years(hrs) } \\
& \text { Age } 5 \text { years(hrs) } \\
& \text { Nap duration }
\end{aligned}
$$

Long-Sleep (LS, $\geq 11 \mathrm{hrs} 30 /$ night)

Age 2 years(hrs)

Age 3 years(hrs)

Age 5 years(hrs)

$\begin{array}{cc}\% & \mathbf{N} \\ 45.5 & 465\end{array}$

Mean (SD)

$\begin{array}{ll}10.0 & 103 \\ 60.0 & 715 \\ 30.0 & 306\end{array}$

$14.5(2.4)$

$14.9 \quad 152$

$6.1 \quad 62$

19.9

203

$30.2(4.6)$

$46.1 \quad 471$

$46.5 \quad 475$

$39.3(1.7)$

$3.4(3.8)$

$72.9 \quad 693$

$20.9 \quad 213$

$5.0 \quad 51$

$20.7 \quad 198$

$41.4(40.8)$

2:04 (0:30)

5.0

51

9:46 (0:29)

9:36 (0:26)

$10: 10(0: 23)$

2:06 (0:35)

48.4

10:37 (0:26)

10:35 (0:24)

10:44 (0:40)

2:05 (0:31)

37.0

378

$11: 29(0: 25)$

11:16 (0:23)

11:06(0:22)

2:04 (0:31)

5.3

$12: 29(0: 27)$

10:34 (0:29)

$10: 49(0: 25)$

2:05 (0:34)

4.3

$12: 33(0: 30)$

12:12 (0:29)

$11: 29(0: 24)$ 
${ }^{\mathrm{a}}$ Equivalent in US dollars : <1600\$, [1600\$-3250\$], > 3250\$ per month

${ }^{\mathrm{b}}$ Number of years of schooling starting from first year of primary school e.g. 12 years corresponds to having completed high school

${ }^{\mathrm{c}}$ Center of epidemiologic studies depression scale, cutoff validated in a French population, measured during pregnancy 
Table 2. Prevalence of behavioral problems at age 5-6 years

\begin{tabular}{|c|c|c|c|c|}
\hline & $\begin{array}{c}\text { Full sample } \\
\qquad \begin{array}{c}\mathbf{N}=\mathbf{1 0 2 1} \\
\%(\mathrm{~N})\end{array}\end{array}$ & $\begin{array}{c}\text { Boys } \\
\mathbf{N}=\mathbf{5 5 0} \\
\%(\mathbf{N})\end{array}$ & $\begin{array}{c}\text { Girls } \\
\mathrm{N}=471 \\
\%(\mathrm{~N})\end{array}$ & $\mathbf{p}$ \\
\hline Conduct problems & $26.2(268)$ & $30.0(165)$ & $21.9(103)$ & 0.008 \\
\hline Hyperactivity/inattention & $26.6(272)$ & $32.0(176)$ & $20.4(96)$ & $<0.001$ \\
\hline Emotional symptoms & $21.5(220)$ & $21.1(116)$ & $22.1(104)$ & 0.70 \\
\hline Peer relationship problems & $30.0(306)$ & $30.5(168)$ & $29.3(138)$ & 0.66 \\
\hline Low pro-social behavior & $26.2(268)$ & $30.5(168)$ & $21.2(100)$ & 0.001 \\
\hline
\end{tabular}


Table 3. Adjusted associations between night-sleep duration trajectories and behavior at age 5-6 years.

\begin{tabular}{|c|c|c|c|c|c|c|c|c|c|c|}
\hline & $\begin{array}{c}\begin{array}{c}\text { Conduct } \\
\text { problems }\end{array} \\
\text { aOR }[95 \% \mathrm{CI}]\end{array}$ & $\mathbf{p}$ & $\begin{array}{c}\text { Hyperactivity/ } \\
\text { inattention } \\
\text { aOR }[95 \% \mathrm{CI}]\end{array}$ & $\mathbf{p}$ & $\begin{array}{c}\begin{array}{c}\text { Emotional } \\
\text { symptoms }\end{array} \\
\text { aOR }[95 \% \mathrm{CI}]\end{array}$ & $\mathbf{p}$ & $\begin{array}{c}\text { Peer } \\
\text { relationship } \\
\text { problems } \\
\text { aOR }[95 \% \mathrm{CI}]\end{array}$ & $\mathbf{p}$ & $\begin{array}{c}\text { Low pro-social } \\
\text { behavior } \\
\text { aOR }[95 \% \mathrm{CI}]\end{array}$ & $\mathbf{p}$ \\
\hline \multicolumn{11}{|l|}{ Raw Model } \\
\hline SS & $1.36[0.72-2.57]$ & 0.34 & $2.61[1.41-4.80]$ & 0.002 & $1.41[0.73-2.73]$ & 0.31 & $1.19[0.63-2.24]$ & 0.59 & $1.72[0.94-3.16]$ & 0.08 \\
\hline MLS & $1.10[0.81-1.49]$ & 0.55 & $1.56[1.14-2.13]$ & 0.005 & $1.03[0.74-1.43]$ & 0.86 & $1.12[0.84-1.51]$ & 0.44 & $0.83[0.61-1.13]$ & 0.23 \\
\hline MHS & Reference & & Reference & & Reference & & Reference & & Reference & \\
\hline $\mathrm{CS}$ & $1.37[0.72-2.57]$ & 0.32 & $1.86[1.00-3.45]$ & 0.05 & $1.18[0.60-2.31]$ & 0.62 & $1.41[0.77-2.58]$ & 0.26 & $1.12[0.60-2.10]$ & 0.71 \\
\hline LS & $0.56[0.24-1.31]$ & 0.18 & $0.71[0.30-1.64]$ & 0.42 & $0.59[0.24-1.44]$ & 0.25 & $1.64[0.87-3.13]$ & 0.14 & $1.00[0.50-2.02]$ & 0.99 \\
\hline \multicolumn{11}{|c|}{ Model $1^{\text {a }}$} \\
\hline SS & $1.05[0.51-2.17]$ & 0.89 & $2.57[1.30-5.08]$ & 0.007 & $1.10[0.53-2.29]$ & 0.80 & $1.08[0.54-2.14]$ & 0.83 & $1.22[0.62-2.39]$ & 0.57 \\
\hline MLS & $1.03[0.74-1.44]$ & 0.85 & $1.73[1.24-2.42]$ & 0.001 & $1.03[0.72-1.45]$ & 0.89 & $1.14[0.83-1.55]$ & 0.42 & $0.73[0.53-1.01]$ & 0.06 \\
\hline MHS & Reference & & Reference & & & & Reference & & Reference & \\
\hline CS & $1.64[0.84-3.21]$ & 0.14 & $2.06[1.05-4.02]$ & 0.03 & $1.23[0.61-2.51]$ & 0.56 & $1.23[0.65-2.32]$ & 0.53 & $1.23[0.63-2.38]$ & 0.55 \\
\hline LS & $0.48[0.2-1.19]$ & 0.11 & $0.61[0.25-1.51]$ & 0.29 & $0.51[0.20-1.3]$ & 0.16 & $1.55[0.79-3.04]$ & 0.21 & $1.04[0.50-2.17]$ & 0.91 \\
\hline \multicolumn{11}{|l|}{ Model $2^{\mathrm{b}}$} \\
\hline SS & $0.88[0.42-1.85]$ & 0.73 & $2.65[1.29-5.45]$ & 0.008 & $1.11[0.53-2.32]$ & 0.79 & $1.06[0.53-2.11]$ & 0.86 & .. & .. \\
\hline MLS & $1.01[0.72-1.42]$ & 0.94 & $1.71[1.20-2.43]$ & 0.003 & $1.03[0.72-1.47]$ & 0.86 & $1.13[0.83-1.55]$ & 0.43 & .. & .. \\
\hline MHS & Reference & & Reference & & Reference & & Reference & & & \\
\hline CS & $1.90[0.95-3.76]$ & 0.07 & $2.03[1.01-4.08]$ & 0.05 & $1.43[0.70-2.92]$ & 0.33 & $1.23[0.65-2.33]$ & 0.52 & .. & .. \\
\hline LS & $0.51[0.20-1.28]$ & 0.15 & $0.67[0.26-1.75]$ & 0.42 & $0.48[0.19-1.22]$ & 0.12 & $1.55[0.79-3.05]$ & 0.20 & .. & .. \\
\hline
\end{tabular}

aOR adjusted odds ratio and 95\%CI

SS Short-Sleep duration trajectory, always <10hrs30/night (5.0\%); MLS Medium-Low-Sleep duration trajectory, 10hrs30-11hrs00/night (48.4\%); MHS Medium-High-Sleep duration trajectory, around $11 \mathrm{hrs} 30 /$ night (37.0\%); LS Long-Sleep duration trajectory, $\geq 11 \mathrm{hrs} 30 /$ night (4.3\%); CS Changing-Sleep duration trajectory, i.e. up to age 3 similar to LS and then to MLS (5.3\%).

${ }^{a}$ Adjusted for recruitment center, household income, parental education, mother's age at delivery, mother's depressive and smoking status during pregnancy, gender, birth order, term at birth, breastfeeding duration, parental antecedents of childhood behavior problems, child temperament traits at age 1, child exact age at behavioral assessment, number of hours spent in front of a television at age 2, care arrangement at age 2, maternal working status at age 2, falling asleep with a parent at age 2 , irregular bed-time at age 2, frequent night waking at age 2 .

${ }^{\mathrm{b}}$ Adjusted on the same variables than model 1, and additionally on the corresponding behavioral measure at baseline (i.e. age 2) 
Table 4. Adjusted associations between night-sleep duration trajectories and hyperactivity/inattention at age 5-6 years by gender.

\begin{tabular}{|c|c|c|c|c|c|}
\hline & & \multicolumn{2}{|c|}{ Boys } & \multicolumn{2}{|c|}{ Girls } \\
\hline & & aOR $[95 \% \mathrm{CI}]$ & $\mathbf{p}$ & aOR [95\% CI] & $\mathbf{p}$ \\
\hline \multicolumn{6}{|l|}{ Model $1^{\mathrm{a}}$} \\
\hline & SS & $2.67[1.11-6.45]$ & 0.03 & $2.35[0.63-8.76]$ & 0.20 \\
\hline & MLS & $1.95[1.23-3.11]$ & 0.005 & $1.44[0.83-2.49]$ & 0.20 \\
\hline & MHS & Reference & & Reference & \\
\hline & $\mathrm{CS}$ & $0.75[0.23-2.48]$ & 0.64 & $2.86[1.16-7.07]$ & 0.02 \\
\hline & LS & $1.25[0.4-3.93]$ & 0.71 & $0.19[0.02-1.51]$ & 0.12 \\
\hline \multicolumn{6}{|l|}{ Model $2^{b}$} \\
\hline & SS & $2.69[1.18-6.16]$ & 0.02 & $2.46[0.63-9.56]$ & 0.19 \\
\hline & MLS & $1.95[1.25-3.03]$ & 0.003 & $1.37[0.78-2.43]$ & 0.28 \\
\hline & MHS & Reference & & Reference & \\
\hline & $\mathrm{CS}$ & $0.76[0.24-2.39]$ & 0.64 & $2.79[1.09-7.17]$ & 0.03 \\
\hline & LS & $1.1[0.37-3.27]$ & 0.87 & $0.2[0.02-1.74]$ & 0.15 \\
\hline
\end{tabular}

aOR adjusted odds ratio and 95\% CI

SS Short-Sleep duration trajectory, always <10hrs30/night (5.0\%); MLS Medium-Low-Sleep duration trajectory, 10hrs30-11 hrs00/night (48.4\%); MHS Medium-High-Sleep duration trajectory, around 11hrs30/night (37.0\%);

LS Long-Sleep duration trajectory, $\geq 11 \mathrm{hrs30/night} \mathrm{(4.3 \% );} \mathrm{CS} \mathrm{Changing-Sleep} \mathrm{duration} \mathrm{trajectory,} \mathrm{i.e.} \mathrm{up} \mathrm{to} \mathrm{age}$ 3 similar to LS and then to MLS $(5.3 \%)$.

${ }^{a}$ Adjusted for recruitment center, household income, parental education, mother's age at delivery, mother's depressive and smoking status during pregnancy, gender, birth order, term at birth, breastfeeding duration, parental antecedents of childhood behavior problems, child temperament traits at age 1 child exact age at behavioral assessment, number of hours spent in front of a television at age 2, care arrangement at age 2 , maternal working status at age 2 , falling asleep with a parent at age 2 , irregular bed-time at age 2 , frequent night waking at age 2 .

${ }^{\mathrm{b}}$ Adjusted on the same variables than model 1, and additionally on the corresponding behavioral measure at age 2 


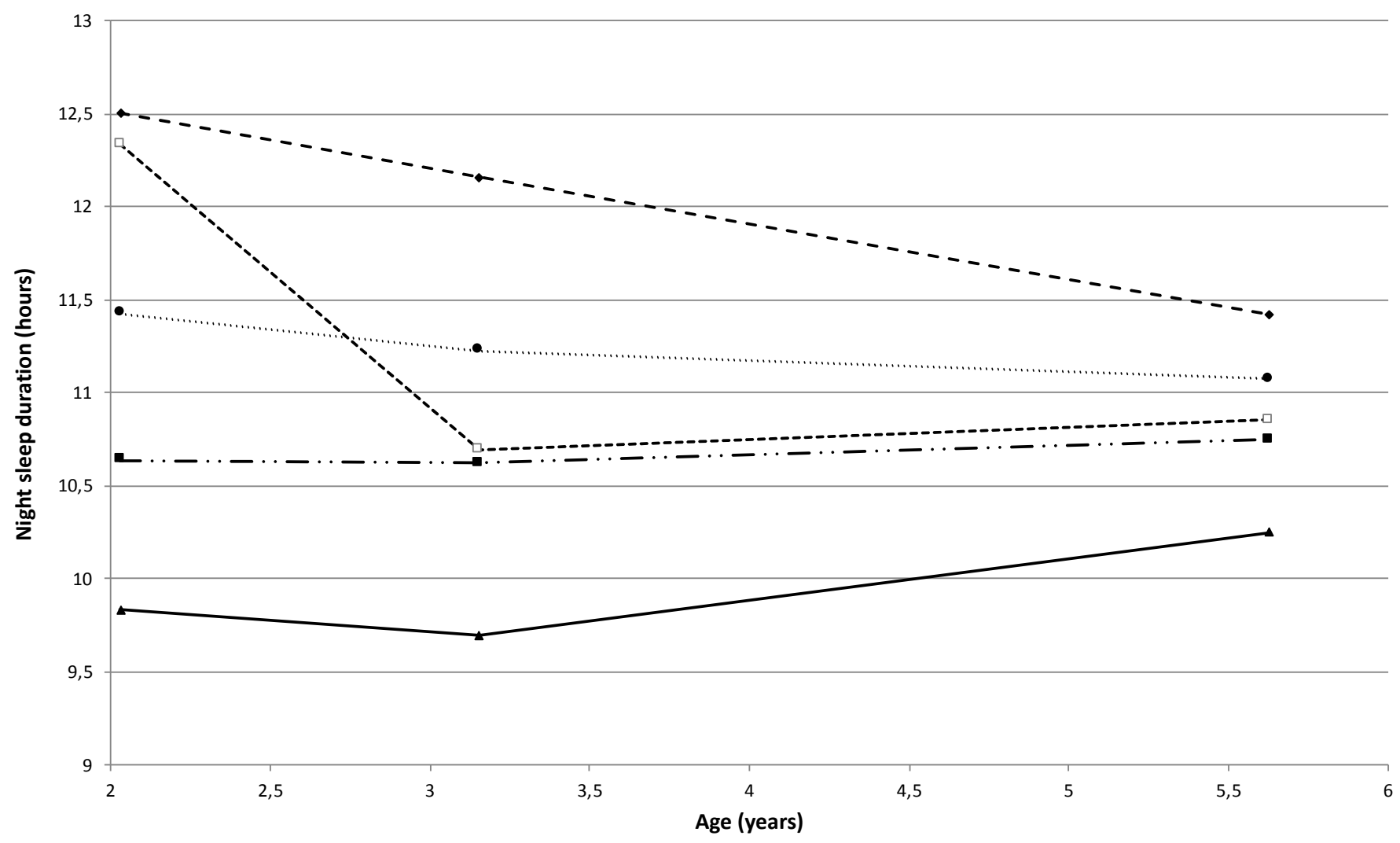

Figure 1. 